\title{
Weiterentwicklungen in der Verhaltenstherapie: Unüberschaubar und identitätszerstörend?
}

\author{
Ulrike Ehlert
}

Klinische Psychologie und Psychotherapie, Psychologisches Institut, Universität Zürich, Schweiz

Als Psychotherapeutinnen und Psychotherapeuten laufen wir zurzeit Gefahr, in der Flut neuer therapeutischer Methoden in «Unkenntnis» zu ertrinken, uns nicht «up to date» zu fühlen und entsprechend verunsichert zu sein. Zumindest sind seit geraumer Zeit in Fachdiskussionen oder Gesprächen mit (fach)politischen Entscheidungsträgern Tendenzen zu verspüren, die darauf hinweisen, dass eine postgraduale Weiterbildung in einem wissenschaftlichen Psychotherapieverfahren nicht mehr ausreichend zu sein scheint. Es gibt nun Weiterbildungsangebote, die schon im Namen darauf hinweisen, dass sie (anscheinend) explizit auf psychologischen Inhalten basieren (wie die psychologische Psychotherapie) oder «Zwei in Einem» vermitteln wie die kognitive Verhaltenstherapie mit verhaltensmedizinischem Schwerpunkt, die kognitive Verhaltenstherapie mit interpersonalem Schwerpunkt, die kognitive Verhaltenstherapie mit systemischem Schwerpunkt. Dann gibt es neue Therapiemethoden, aus deren Namen psychotherapeutische Kolleginnen und Kollegen, die sich weniger up to date fühlen, nicht so genau erschließen können, welcher Therapierichtung diese Verfahren zugeordnet werden sollen. Beispiele dafür sind Schematherapie (Verhaltenstherapie oder Psychoanalyse?) oder Training emotionaler Kompetenz (Verhaltenstherapie oder Gesprächstherapie?) oder Mindfulness (Verhaltenstherapie oder Buddhismus?).

Alle jene deutschsprachigen Psychotherapeutinnen und Psychotherapeuten, die in den 70er und 80er Jahren des 20. Jahrhunderts eine (damals oft noch rudimentär organisierte curriculare) Verhaltenstherapieweiterbildung absolviert haben, haben schon einige «Verhaltenstherapie-Wenden» und damit mehr oder weniger stark ausgeprägte Verunsicherungen hinter sich. Von der lernpsychologischen zur kognitiven, weiter zur ressourcenorientierten Verhaltenstherapie usw. Auch die Anwendungsgebiete der Verhaltenstherapie haben sich deutlich erweitert: von der Pädagogik und Psychotherapie (im engeren Sinn) hin zu betriebswirtschaftlichen Feldern, zur Arbeit mit Kindern oder zur Medizin, wie es das Beispiel der Verhaltensmedizin zeigt. Trotz aller Erweiterungen und Trendwenden halten sich viele Verhaltenstherapeuten aber doch noch für Verhaltenstherapeuten, wobei man Viktor Frankl [1997, p 11] durchaus zustimmen kann: «Jede Zeit hat ihre Neurose - und jede Zeit braucht ihre Psychotherapie.» Die enge Orientierung der ursprünglichen Verhaltenstherapie an lerntheoretischen Konzepten und die fehlende Berücksichtigung organismusbezogener Variablen sind historisch zu sehen und als Kontrapunkt zu psychoanalytischen Konzepten zu verstehen - eben ein Resultat jener Zeit. Es wäre erschreckend, wenn Forschung, die materiell und immateriell kostenintensiv ist, keinen Erkenntnisfortschritt brächte und sich die Anwendungsfelder eines erfolgreichen theoretischen Grundkonzeptes nicht erweiterten.

Nehmen wir das Beispiel der Persönlichkeitsstörungen. Unter der historischen Perspektive und gemäß den lerntheoretischen Grundkonzepten waren Persönlichkeitsstörungen lange Zeit kein Thema in der Verhaltenstherapie. Klassisch verhaltenstherapeutische Erfahrungen mit persönlichkeitsgestörten Patientinnen und Patienten ließen sich auf Therapeutenseite häufig mit dem Satz zusammenfassen: Irgendwie läuft die Therapie überhaupt nicht, wie sie soll. Neue Therapieentwicklungen, basierend auf verhaltenstherapeutischen Grundannahmen (wie die dialektisch-behaviorale Therapie [Linehan, 1996] oder die Schematherapie [Young et al., 2008] tragen den spezifischen Bedürfnissen dieser Patientinnen und Patienten differenzierter Rechnung. Auch erfahrene Verhaltenstherapeutinnen und -therapeuten gestehen sich und dieser Patientengruppe unter anderem eine längere Therapiedauer und vielleicht eine größere Geduld zu. Darüber hinaus werden sich gute Therapeutinnen und Therapeuten auch über wirksame Therapiemethoden, die auf bestehendes (verhaltenstherapeutisches) Wissen aufbauen, informieren und diese erlernen. Von einem Chirurgen oder einer Chirurgin erwar-

\section{KARGER}

Fax +497614520714

Information@Karger.de

www.karger.com (c) 2009 S. Karger GmbH, Freiburg

Accessible online at:

www.karger.com/ver 
ten wir ja auch, dass sie oder er nicht mehr den kompletten Bauchraum öffnet, wenn es für den gleichen Eingriff minimal invasive Alternativen gibt; diese neuen Methoden sind aber immer noch chirurgische Methoden! Möglicherweise könnte es aber auch im chirurgischen Fall sinnvoll sein, operative Maßnahmen mit einer weiteren Therapie zu kombinieren (z.B. Medikamentengabe). Bis zu einem gewissen Grad kann das chirurgische Fachpersonal diese zusätzlichen Therapiemaßnahmen übernehmen, für bestimmte Spezialmaßnahmen muss eine anderer Fachkollege bzw. -kollegin herangezogen werden. Ich denke, dieses Beispiel lässt sich auf unsere Profession übertragen.

Es ist wichtig, dass zu Beginn einer postgradualen Weiterbildung die theoretischen Konzepte einer Therapierichtung differenziert vermittelt werden und die theoriegeleiteten therapeutischen Techniken erlernt und trainiert werden. Wenn diese Basis gegeben ist, lassen sich z.B. im Rahmen der Fortbildung weitere, auch Verhaltenstherapie-unabhängige Verfahren und Therapierichtungen erlernen, wie spezielle Entspannungstechniken, sexualtherapeutische Verfahren oder paartherapeutische Interventionen. Aber eine identitätsstiftende Wissens- und Erfahrungsbasis in einem Therapieverfahren sollte am Beginn einer erfolgreichen und persönlich zufriedenstellenden Therapeutenkarriere stehen. Die «Kombinationspackung», die sich aus der Wissensvermittlung von zwei Therapieschulen in der gleichen Zeit wie von einer Therapieschule zusammensetzt, kann nach meiner persönlichen Einschätzung nicht funktionieren bzw. müsste erst einmal empirisch geprüft werden.

Im Pro und Contra dieses Heftes haben sich zwei Verhaltenstherapeuten zu dem von mir skizzierten Thema geäußert. Sie haben ausführliche Plädoyers zu der Frage dargelegt, ob wir noch Psychotherapieschulen brauchen. Beide, Prof. Dr. Mathias Berger und Prof. Dr. Michael Linden, sind Wissenschaftler und praktisch arbeitende Verhaltenstherapeuten - und vertreten dennoch recht unterschiedliche Standpunkte. In jedem Fall ist das Pro und Contra dieser Ausgabe der Verhaltenstherapie lesenswert, um die eigene Position zu hinterfragen, zu klären und möglicherweise Konsequenzen für die eigene Weiter- und Fortbildung zu ziehen.

\section{Literatur}

Frankl V: Das Leiden am sinnlosen Leben. Psychotherapie für Heute. Freiburg, Herder, 1997.

Linehan M: Dialektisch-Behaviorale Therapie der Borderline-Persönlichkeitsstörung. München, CIP-Medien, 1996.

Young JE, Klosko JS, Weishaar ME: Schematherapie. Ein praxisorientiertes Handbuch, ed 2. Paderborn, Junfermann, 2008. 